\title{
Limits of Inner Superposition Operators and Young Measures
}

\author{
L. De Pascale
}

\begin{abstract}
We apply some well known theorems from the theory of Young measures to the theory of inner superposition (composition) operators. We give an explicit characterization of the limit operator of a weak-convergent sequence of inner superposition operators between Lebesgue spaces.
\end{abstract}

Keywords: Inner superposition operators, weak limits of operator sequences

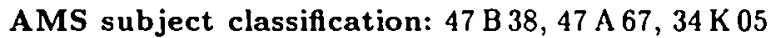

\section{Introduction}

In order to study the stability of the solution of boundary data problems for functionaldifferential equations one is required to study weak and strong convergence (see Section 2 for the definition) of operators [1], while the applications to optimal control problems governed by such equations require the study of the so-called weak-continuous convergence (see Section 2 for the definition and Section 4 and [3] for a full explanation).

In the case of functional-differential equations with deviated argument, inner superposition operators are naturally involved. Let $\Omega$ be an open subset of $\mathbb{R}^{N}$ and let $g: \Omega \rightarrow \mathbb{R}^{N}$ be a function such that

$$
|B|=0 \quad \Longrightarrow \quad\left|g^{-1}(B)\right|=0
$$

where $B$ is a subset of $\Omega$ and $|B|$ is the Lebesgue measure of the set. The inner superposition operator associated to $g$ is defined by

$$
\left(T_{g} u\right)(x)= \begin{cases}u(g(x)) & \text { if } g(x) \in \Omega \\ 0 & \text { if } g(x) \notin \Omega .\end{cases}
$$

Since (1) holds, this operator is well defined in the space of all measurable functions on $\Omega$. Indeed, (1) guarantees that the value of the operator does not depend on the representative in the equivalence class of functions.

Young measures have been introduced (by L. C. Young) in the Calculus of Variations as generalized solutions for various kinds of problems, and are now used for many

L. De Pascale: Univ. di Pisa, Dept. Math., via Buonarroti 2, 56127 Pisa, Italy depascal@gauss.dm.unipi.it 
different applications (see $[19,20]$ and the references therein). The theory developed up to now is fairly big and its discussion or exposition are out of the targets of this paper. In the next section we just report the basic definitions and theorems that we will need in this paper.

In this paper we first use Young measures to give a representation formula for the limit of a sequence of inner superposition operators. Then, in the last section, we give some application to optimal control problems governed by a non-local state equation.

\section{Notation and preliminary results}

In this section we set the notation, and recall the main results about convergence of sequences of operators, in particular about inner superposition operators. From now on, $\Omega$ is an open bounded subset of $\mathbb{R}^{N}$ and $g_{k}$ a function verifying (1). As usual, $\chi_{B}$ is the indicatrix of the set $B$ (i.e. is equal to 1 on $B$ and 0 outside $B$ ). Further, $\mathcal{L}^{N}$ is the Lebesgue measure in $\mathbb{R}^{N}$, and $\mathcal{L}^{N}(B)$ is sometimes denoted by $|B|$. The derivative of a measure with respect to another will be intended in Radon-Nikodym sense (see [13]). $\mathcal{M}\left(\Omega, \mathbb{R}^{N}\right)$ is the space of vector-valued Radon measures on $\Omega$, which is known to be the dual space of $C_{0}(\Omega)$ in the case $N=1$, and $\mathcal{M}^{+}\left(\Omega \times \mathbb{R}^{N}\right)$ is the space of positive Radon measures on $\Omega \times \mathbb{R}^{N}$.

Unless otherwise explicitly mentioned, we always assume $A_{\nu}, A: X \rightarrow Y$ to be linear operators between Banach spaces $X$ and $Y$. We indicate with $X^{\prime}$ and $Y^{\prime}$ the duals of $X$ and $Y$, respectively, and with $A_{\nu}^{\prime}, A^{\prime}: Y^{\prime} \rightarrow X^{\prime}$ the adjoints of the operators $A_{\nu}, A$, respectively. The space of linear operators between $X$ and $Y$ will be denoted by $\mathcal{L}(X, Y)$, and $\|T\|_{p \rightarrow q}$ will be the norm of the operator $T$ in the space $\mathcal{L}\left(L^{p}, L^{q}\right)$.

Definition 2.1. We say that the sequence $A_{\nu}$ converges to $A$

(U) uniformly, if it converges in the norm of $\mathcal{L}(X, Y)$

(S) strongly, if $A_{\nu} x \rightarrow A x$ in $Y$ for all $x \in X$

(W) weakly, if $A_{\nu} x \rightarrow A x$ in $Y$ for all $x \in X$

(CW) continuously weakly, if $A_{\nu} x_{\nu} \rightarrow A x$ in $Y$ for any weakly converging sequence $x_{\nu}-x$ in $X$.

For the weak and continuous weak convergence we adopt the notation $A_{\nu}-A$ and $A_{\nu} \stackrel{(\underline{c w)})}{\rightarrow} A$, respectively. We recall that all the above types of convergence are induced by the respective topologies in $\mathcal{L}(X, Y)$, which we will refer to as uniform, strong, weak and continuous weak operator topologies, respectively, but in general only the uniform topology is metrizable (see [12: Chapter IV]). In this paper, however, we operate in terms of sequences. $\left(U^{\prime}\right),\left(S^{\prime}\right)$ and $\left(W^{\prime}\right)$ denote respectively the uniform, strong and weak (pointwise) convergences of the sequence $A_{\nu}^{\prime}$ to $A^{\prime}$.

In the next lemma we summarize the relationships among various kinds of operator convergences.

Lemma 2.1. The following implications hold:

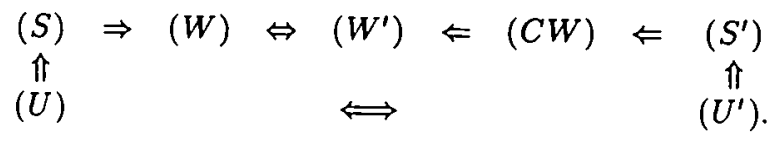


Moreover, if $X$ is reflexive and $Y$ is uniformly convex (in particular, if $Y$ is a Hilbert space), then $(C W) \Leftrightarrow\left(S^{\prime}\right)$. Any of the above convergences implies the uniform boundedness of the whole sequence and the boundedness of the limit operator.

Proof. For the proof of most of the implications the reader may consult [11], the others being trivial

Remark. All the convergences of the previous lemma imply the weak convergence, so a representation theorem for weak limits cover all the others cases.

2.1 Convergence of inner superposition operators. We now turn our attention to the case of inner superposition operators. Inner superposition operators have been introduced in [1] while the study of strong convergence of sequences of these operators has been started by M. E. Drakhlin [9] in order to enable the study of continuous dependence on parameters of solutions of functional differential equations. The interest on weak continuous convergence in optimal control theory is explained in $[3,4]$ for the case of inner superposition operators. We recall some results contained in [11], the first important paper on the subject, and from [15].

Let $\widetilde{\mathcal{M}}(\Omega)$ the space of measurable functions on $\Omega$.

Theorem 2.1. Let a sequence of inner superposition operators $T_{g^{\nu}}: \widetilde{\mathcal{M}} \rightarrow \widetilde{\mathcal{M}}$ converge strongly to an operator $T: \widetilde{\mathcal{M}} \rightarrow \widetilde{\mathcal{M}}$. Then $T$ is an inner superposition operator, that is $T=T_{g}$ for some $g$.

Theorem 2.2. Let a sequence of inner superposition operators $T_{g^{\nu}}: L^{p} \rightarrow L^{q}$ converge weakly to an operator $T_{g}: L^{p} \rightarrow L^{q}$, while $1 \leq q \leq p<+\infty$. Then the convergence is strong.

Theorem 2.3. Let a sequence of inner superposition operators $T_{g^{\nu}}: L^{p} \rightarrow L^{q}$ converge weakly to an operator $T: L^{p} \rightarrow L^{q}$, while $1 \leq q \leq p<+\infty$. Then $T$ has the following properties:

(i) $T$ is a positive operator (i.e. maps non-negative functions in non-negative ones).

(ii) $\|T\|_{\infty \rightarrow r} \leq|\Omega|^{\frac{1}{r}}$, where $1 \leq r \leq+\infty$.

(iii) $T \in \mathcal{L}\left(L^{r}, L^{s}\right)$, if either $1 \leq s \leq r<+\infty$ and $\frac{r}{s} \geq \frac{p}{q}$, or $r=+\infty$ and $1 \leq s \leq+\infty$.

Finally, we recall a recent result from [15] which states that the linear combinations of inner superposition operators are sequentially dense in $\mathcal{L}\left(L^{p}, L^{q}\right)$ with respect to the strong and weak-continuous convergences.

Theorem 2.4. Any operator in $\mathcal{L}\left(L^{p}, L^{q}\right)(1<q \leq p<+\infty)$ is a limit of some sequence of linear combinations of inner superposition operators, converging both strongly and continuously weakly.

2.2 Young measures theory. In this short subsection we recall the main definitions and results of Young measures theory. Moreover, we give some fundamental bibliographical references on this subject. This introduction is in the spirit of [7]. 
Definition 2.2. Let $\Omega$ a bounded open set in $\mathbb{R}^{N}$, and let $u \in L^{\infty}\left(\Omega, \mathbb{R}^{N}\right)$. The measure $\mathcal{Y}_{u} \in \mathcal{M}^{+}\left(\Omega \times \mathbb{R}^{N}\right)$ defined by

$$
\int_{\Omega \times \mathbb{R}^{N}} \varphi d \mathcal{Y}_{u}=\int_{\Omega} \varphi(x, u(x)) d x
$$

for all $\varphi: \Omega \times \mathbb{R}^{N} \rightarrow \mathbb{R}, \varphi \in C_{b}^{0}\left(\Omega \times \mathbb{R}^{N}\right)$, is called Young measure associated to $u$.

It follows from this definition that

$$
\pi_{\#} \mathcal{Y}_{u}=\mathcal{L}^{N}\lfloor\Omega
$$

where $\pi$ denote the projection $\Omega \times \mathbb{R}^{N} \rightarrow \Omega$ and $\pi_{\#} \mu(E):=\mu\left(E \times \mathbb{R}^{N}\right)$. The last property motivates the following definition.

Definition 2.3. A Young measure in $\Omega \times \mathbb{R}^{N}$ is a non-negative Radon measure $\mu \in \mathcal{M}\left(\Omega \times \mathbb{R}^{N}\right)$ satisfying $\pi_{\# \mu}=\mathcal{L}^{N}\lfloor\Omega$.

Then a standard application of the disintegration theorem for measures allow us to write a Young measure as a parametrized measure, i.e. a weakly measurable function $\nu: \Omega \rightarrow \mathcal{M}\left(\mathbb{R}^{N}\right)$. We will use for such a function the notation $\nu=\left\{\nu_{x}\right\}_{x \in \Omega}$.

The fundamental theorem for Young measures has various versions more or less general. We report the following from [2].

Theorem 2.5 (see Ball [2]). Let $\Omega$ be a bounded measurable set in $\mathbb{R}^{N}$ and let $K \subset \mathbb{R}^{N}$ be closed. Let $\left\{u_{k}\right\}$ be a sequence of measurable maps with values in $\mathbb{R}^{N}$ satisfying for any open set $U$ containing $K$

$$
\mathcal{L}^{N}\left(\left\{x \in \Omega \mid u_{k}(x) \notin U\right\}\right) \rightarrow 0 \quad \text { as } k \rightarrow \infty
$$

Then there exist a subsequence $\left\{u_{k_{i}}\right\}$ of $\left\{u_{k}\right\}$ and a measurable family of positive measures $\left\{\nu_{x}\right\}_{x \in \Omega}$ on $\mathbb{R}^{N}$ such that

(i) $\nu_{x}\left(\mathbb{R}^{N}\right) \leq 1$ for a.e. $x \in \Omega$

(ii) $\operatorname{supp} \nu_{x} \subset K$ for a.e. $x \in \Omega$

(iii) for any $\psi \in C^{0}\left(\mathbb{R}^{N}\right)$ with $\psi(y) \rightarrow 0$ as $|y| \rightarrow+\infty$ we have $\psi$ o $u_{k_{i}} \rightarrow$ v weakly* in $L^{\infty}$ where $v(x)=\int_{\mathbb{R}^{N}} \psi(y) d \nu_{x}(y)$.

Moreover, if we assume that $\sup _{k} \int_{\Omega} h\left(\left|u_{k}\right|\right) d x<\infty$ for some continuous non-decreasing function $h:[0, \infty] \rightarrow \mathbb{R}$ such that $h(t) \rightarrow \infty$ when $t \rightarrow \infty$, then $\nu_{x}$ is a probability measure.

For a short and smart introduction of the concept of Young measure the reader is referred to [7] and to $[2,16-20]$ and references therein for a deeper account on old and recent development and application of the theory. 


\section{A representation theorem}

Let $\left\{T_{g_{n}}\right\}$ be a sequence of inner superposition operators in $\mathcal{L}\left(L^{p}(\Omega), L^{q}(\Omega)\right.$ ), and let $x_{0} \notin \Omega$ be fixed. We can assume that for $x \in \Omega$ either $g_{n}(x) \in \Omega$ or $g_{n}(x)=x_{0}$ without modifying the operators $T_{g_{n}}$.

Assume that $T_{g_{n}} \rightarrow A$ weakly. The scope of this section is to give a representation formula for $A$.

Lemma 3.1. Assume that $\left\{g_{n}\right\} \subset L^{\infty}\left(\Omega, \mathbb{R}^{N}\right)$ satisfy all the hypotheses above, i.e. the values of $g_{n}(x)$ are in $\Omega \bigcup\left\{x_{0}\right\}$, and that the associate sequence of inner superposition operators converges weakly to some operator $A$. Then:

a) $\left\{g_{n}\right\}$ generates just one Young measure $\nu=\left\{\nu_{x}\right\}_{x \in \Omega}$.

b) For a.e. $x, \operatorname{supp}\left(\nu_{x}\right) \subset \Omega \bigcup\left\{x_{0}\right\}$.

c) If $\varphi \in C_{0}(\Omega)$, then $A \varphi(x)=\left\langle\varphi, \nu_{x}\right\rangle$.

Proof. Let $u \in C_{0}\left(\mathbb{R}^{N}\right)$ and let $\vec{u}$ be the restriction of $u$ to $\Omega$. We have

$$
\begin{aligned}
u\left(g_{n}(x)\right) & =\left(T_{g_{n}} \bar{u}\right)(x)+\chi_{\Omega \backslash g_{n}^{-1}(\Omega)}(x) u\left(x_{0}\right) \\
& =\left(T_{g_{n}} \bar{u}\right)(x)+\left(\chi \Omega-\chi_{g_{n}^{-1}(\Omega)}\right)(x) u\left(x_{0}\right) \\
& =\left(T_{g_{n}} \bar{u}\right)(x)+\left(\chi \Omega-T_{g_{n}} \chi \Omega\right)(x) u\left(x_{0}\right) \\
& \rightarrow A \bar{u}(x)+(\chi \Omega-A \chi \Omega)(x) u\left(x_{0}\right) .
\end{aligned}
$$

So the weak limit in $L^{q}$ of $u\left(g_{n}\right)$ exists and does not depend on the subsequence of $\left\{g_{n}\right\}$ we choose. This proves statement(a). Statement (b) follows from the fundamental theorem on Young measures, while statement (c) follows from the proof of statement

(a)

It is natural now to define an operator $\tilde{A}: C_{0}(\Omega) \rightarrow L^{q}(\Omega)$ in the following way:

$$
(\tilde{A} u)(x)=\left\langle u, \nu_{x}\right\rangle \text {. }
$$

Using the Jensen inequality and the fact that $\tilde{A}$ coincides with the restriction of $A$ to $C_{0}(\Omega)$ we obtain

$$
\begin{aligned}
\|\tilde{A} u(x)\|_{L^{q}}^{q} & =\int_{\Omega}\left|\left\langle u, \nu_{x}\right\rangle\right|^{q} d x \\
& \leq \int_{\Omega}\left\langle|u|^{q}, \nu_{x}\right\rangle d x \\
& =\lim _{n \rightarrow \infty} \int_{\Omega} T_{g_{n}}\left(|u|^{q}\right)(x) d x \\
& \leq c\|u\|_{L^{p}}^{q}
\end{aligned}
$$

for any $u \in C_{0}(\Omega)$.

The following theorem adds a "nice" property to the parametrized measure $\nu=$ $\left\{\nu_{x}\right\}_{x \in \Omega}$. For each Borel set $B \subset \Omega$ define

$$
\tilde{\mu}(B)=\int_{\Omega} \nu_{\mathbf{x}}(B) d x
$$

This is a measure on $\Omega$. 
Theorem 3.1. If the operator $\tilde{A}$ admits a continuous extension in $\mathcal{L}\left(L^{p}, L^{q}\right)$, then $\tilde{\mu}$ is absolutely continuous with respect to the Lebesgue measure $\mathcal{L}^{N}$.

Proof. Suppose by contradiction that there exists $\varepsilon>0$ such that for any $\delta>0$ there exists an open set $A_{\delta}$ with $\left|A_{\delta}\right|<\delta$ but $\tilde{\mu}\left(A_{\delta}\right)>\varepsilon$. Then we can choose a function $\varphi_{\delta} \in C_{0}\left(\mathbb{R}^{N}\right)$ such that $\varphi_{\delta} \equiv 1$ on $A_{\delta}, 0 \leq \varphi_{\delta} \leq 1, \varphi_{\delta}\left(x_{0}\right)=0$ and $\left\|\varphi_{\delta}\right\|_{L^{p}} \leq 2 \delta$. Then we have

$$
\int_{\Omega}\left\langle\varphi_{\delta}, \nu_{x}\right\rangle^{q} d x \geq \int_{\Omega}\left(\nu_{x}\left(A_{\delta}\right)\right)^{q} d x \geq c \tilde{\mu}\left(A_{\delta}\right)^{q} \geq c \varepsilon^{q} .
$$

Then $\varphi_{\delta} \rightarrow 0$ in $L^{p}$ but $A \varphi_{\delta} \not 0$ in $L^{q}$ which is a contradiction

Consider now a positive Borel function $h$. For each $x \in \Omega$ the integral

$$
\left\langle h, \nu_{x}\right\rangle
$$

is well defined. Moreover, thanks to Theorem 2.5 given another Borel function $h_{1}$ such that $h_{1}=h \mathcal{L}^{N}$-a.e., we have

$$
\left\langle h, \nu_{x}\right\rangle=\left\langle h_{1}, \nu_{x}\right\rangle \quad \mathcal{L}^{N}-\text { a.e. }
$$

For any positive $u \in L^{p}(\Omega)$ we can define $(\tilde{A} u)(x)=\left\langle\tilde{u}, \nu_{\dot{x}}\right\rangle$, where $\tilde{u}$ is any Borel representative of $u$. Let us now prove that $\tilde{A}=A$ on the cone of the positive functions of $L^{p}$. This will permit to define $\tilde{A} u=\tilde{A} u^{+}-\tilde{A} u^{-}$and to prove that

$$
A u(x)=\left\langle u, \nu_{x}\right\rangle
$$

Theorem 3.2. For all positive $u \in L^{p}, A u=\tilde{A} u$.

Proof. To prove that the two operators coincide we will prove that there exists a constant $c$ such that for all positive functions $u$ in $L^{p}$ the inequality $\|\tilde{A} u\|_{L^{q}} \leq c\|u\|_{L^{p}}$ holds true. From this we will reach the conclusion, since we know that $\tilde{A}$ and $A$ coincide on a dense subset, namely $C_{0}(\Omega)$, and that $A$ is continuous.

Step 1 (Bounded functions): Let $0 \leq h(x) \leq M, \varepsilon>0$, and consider a function $h_{\varepsilon} \in C_{0}(\Omega)$ such that $\mathcal{L}^{N}\left(\left\{x \in \Omega \mid h(x) \neq h_{\varepsilon}(x)\right\}\right) \leq \varepsilon,\left\|h_{\varepsilon}-h\right\|_{L^{p}} \leq \varepsilon$ and $0 \leq h_{\varepsilon} \leq 2 M$. We get

$$
\int_{\Omega}\left\langle h, \nu_{x}\right\rangle^{q} d x \leq \int_{\Omega}\left\langle h(x)^{q}, \nu_{x}\right\rangle d x=\int_{\Omega}\left\langle h_{\varepsilon}^{q}, \nu_{x}\right\rangle d x+\int_{\Omega}\left\langle h^{q}-h_{\varepsilon}^{q}, \nu_{x}\right\rangle d x .
$$

For the first term in the last expression we have

$$
\begin{aligned}
\int_{\Omega}\left\langle h_{\varepsilon}^{q}, \nu_{x}\right\rangle d x & =\lim _{n \rightarrow \infty} \int_{\Omega} T_{g_{n}}\left(h_{\varepsilon}^{q}\right)(x) d x \\
& =\lim _{n \rightarrow \infty} \int_{\Omega^{\prime}}\left(T_{g_{n}} h_{\varepsilon}\right)^{q}(x) d x \\
& =\lim _{n \rightarrow \infty}\left\|T_{g_{n}} h_{\varepsilon}\right\|_{L^{q}}^{q} \\
& \leq c^{q}\left\|h_{\varepsilon}\right\|_{L^{p}}^{q}
\end{aligned}
$$


and for the second term

$$
\int_{\Omega}\left\langle h_{\varepsilon}^{q}-h^{q}, \nu_{x}\right\rangle d x \leq \int_{\Omega} \int_{\Omega \backslash \Omega_{\varepsilon}} 2 M d \nu_{x} d x=2 M \tilde{\mu}\left(\Omega \backslash \Omega_{\varepsilon}\right)
$$

where $\Omega_{\varepsilon}$ denotes the set $\left\{x \in \Omega \mid h(x) \neq h_{\varepsilon}(x)\right\}$. Since $\tilde{\mu}$ is absolutely continuous with respect to $\mathcal{L}^{N}$, the proof of this step is completed.

Step 2: $0 \leq h(x) \leq \infty$ (the unbounded case). We apply a classical technique from the theory of semicontinuity for integral functionals. Let $\left\{h_{n}\right\}$ be a pointwise increasing sequence of bounded functions converging almost everywhere and in $L^{p}$ to $h$. Using the Beppo-Levis theorem we get

$$
\left\langle h_{n}, \nu_{x}\right\rangle^{q} \rightarrow\left\langle h, \nu_{x}\right\rangle^{q} \quad \text { a.e. }
$$

and, using the Fatou lemma,

$$
\int_{\Omega}\left\langle h, \nu_{x}\right\rangle^{q} d x \leq \liminf _{n \rightarrow \infty} \int_{\Omega}\left\langle h_{n}, \nu_{x}\right\rangle^{q} d x \leq \liminf _{n \rightarrow \infty} c\left\|h_{n}\right\|_{L^{p}}^{q}
$$

Thus the theorem is proved

\section{Some applications}

Consider a sequence of optimal control problems for abstract state equations of the form

$$
\min \left\{J(u, y): A_{n}(y)=B_{n} u \quad((u, y) \in U \times Y)\right\}
$$

where $Y$ is a topological space of states, $U$ is an Hilbert space of controls, and

$$
J: U \times Y \rightarrow \overline{\mathbb{R}}
$$

is the cost functional defined by

$$
J(u, y)=\frac{1}{2}\|u\|^{2}+\Psi(y)
$$

with $\Psi$ a continuous functional on $Y, A_{n}: Y \rightarrow V$, and $B_{n} \in \mathcal{L}(U, V)$ where $V$ is some reflexive Banach space. A general theory of the convergence of optimal control problems in this setting as been developed in [3].

Assume that $A_{n} \stackrel{G}{\rightarrow} A$ (see [3] for the definition), and that $B_{n} \rightarrow B$ and $B_{n} B_{n}^{*} \rightarrow K$, where $K$ is some linear operator. Then the following theorem holds (see [3]).

Theorem 4.1. Let $\left(u_{n}, y_{n}\right) \in U \times Y$ be a sequence satisfying $A_{n}\left(y_{n}\right)=B_{n} u_{n}$ for every $n \in \mathbb{N}$ and

$$
\lim _{n \rightarrow \infty} J\left(u_{n}, y_{n}\right)=\inf _{U \times Y}\left\{J(u, y): A_{n}(y)=B_{n} u\right\}
$$


(in particular, $\left(u_{n}, y_{n}\right)$ can be an optimal pair for the $n$-th problem). Suppose that $\left(u_{n}, y_{n}\right) \rightarrow(u, y)$ in $U \times Y$. Then:

(i) $(u, y) \in U \times Y$ is an optimal pair for the problem

$$
\min _{U \times Y}\left\{J(u, y)+\frac{1}{2} \inf \left\{\left\langle v^{*}, A(y)-B u\right\rangle: E v^{*}=A(y)-B u \quad\left(v^{*} \in V^{*}\right)\right\}\right\}
$$

where $E=K-B B^{*}$.

(ii) $\lim _{n \rightarrow \infty} J\left(u_{n}, y_{n}\right)=m$, where $m$ is the minimum value of problem (19).

It is clear that the limit problem can be of different nature from those of the sequence (16) (see $[3,4]$ for examples). If $K=B B^{*}$, then the limit problem (19) can be written as

$$
\min \{J(u, y): A(y)=B u\}
$$

For example, this occur if $Y$ is a uniformly convex Banach space and $B_{n} \stackrel{c w}{ } B$ continuously weakly.

We now consider the case of state equations of the form

$$
\left.\begin{array}{rl}
y^{\prime} & =a(t, y(t))+T_{g_{n}} u(t) \quad(t \in[0,1]) \\
y(0) & =y_{0}
\end{array}\right\}
$$

According to our notation we have

$$
\left.\begin{array}{l}
A_{n} y=A y=\left(y^{\prime}-a(t, y(t)), y(0)-y_{0}\right) \\
B_{n} u=\left(T_{g_{n}} u, 0\right)
\end{array}\right\}
$$

Using the results of the previous sections we can state that if the sequence $\left\{T_{g_{n}}\right\}$ converges continuously weakly to some operator, then the limit problem is still an optimal control problem and that the state equation is given by

$$
\left.\begin{array}{rl}
y^{\prime} & =a(t, y(t))+\left\langle\nu_{t}, u\right\rangle \\
y(0) & =y_{0}
\end{array}\right\}
$$

Let us give some examples.

Example 4.1. If the $n$-th state equation is

$$
\left.\begin{array}{rl}
y^{\prime} & =a(t, y(t))+u(m x-[m x]) \\
(0) & =y_{0}
\end{array}\right\}
$$

where $[x]$ is the integer part of $x$, then the limit state equation is

$$
\left.\begin{array}{rl}
y^{\prime} & =a(t, y(t))+\int_{0}^{1} u(s) d s \\
(0) & =y_{0}
\end{array}\right\}
$$


Example 4.2. If the $n$-th state equation is

$$
\left.\begin{array}{rl}
y^{\prime} & =a(t, y(t))+u(\sin (2 k \pi x)) \\
y(0) & =y_{0},
\end{array}\right\}
$$

then the limit state equation is

$$
\left.\begin{array}{rl}
y^{\prime} & =a(t, y(t))+\int_{-1}^{1} \frac{1}{\pi} \frac{1}{\sqrt{1-s^{2}}} u(s) d s \\
y(0) & =y_{0} .
\end{array}\right\}
$$

Note that in both examples we have a sequence of optimal control problems governed by a functional-differential equation with deviating argoument converging to an optimal control problem governed by an integro-differential state equation.

Remark. Note that Theorems 2.1 and 2.2 can be proved by using our results and some elementary theorems on convergence of measures.

Acknowledgements. The author is grateful to the Department of Mathematical Sciences of the Carnegie Mellon University (Pittsburgh) where this paper as been partially written, to Dott. Lorenzo Freddi (University of Udine, Italy) for the numerous corrections in the preliminary version of this paper, and to Doct. M. A. Sychev (Carnegie Mellon University) for many valuable conversations on Young's measures.

\section{References}

[1] Azbelev, N. V., Maksimov, V. P. and L. F. Rakhmatullina: Introduction to the Theory of Linear Functional Differential Equations (in Russian). Moscow: Nauka 1991; Engl. transl.: Atlanta (GA, USA): World Fed. Publ. Comp. 1995.

[2] Ball, J. M.: A version of the fundamental theorem for Young measures. Lect. Notes Phys. 344 (1989), $207-215$.

[3] Buttazzo, G. and L. Freddi: Optimal control problems with weakly converging input operators. Dis. Cont. Dyn. Syst. 1 (1995), $401-420$.

[4] Buttazzo, G., Drakhlin, M. E., Freddi, L. and E. Stepanov: Homogenization of optimal control problems for functional differential equations with deviating argument. J. Optim. Theory Appl. 93 (1997), 103 - 119.

[5] Chistyakov, A. V.: A pathological counterexample for hypothesis of non-Fredholm property in algebras of weighted shift operators (in Russian). Izvestiya Vysh. Uch. Zav. (Matematika) 39 (1955) 10, 76 - 86; English transl. in: Russ. Math. 39 (1995) 10, 73 - 83.

[6] Drakhlin, M. E. and E. Stepanov: $\Gamma$-convergence for a class of functionals with deviating argument. J. Conv. Anal. 4 (1997), $69-89$.

[7] Giaquinta, M., Modica, G. and J. Soucek: Cartesian Currents in the Calculus of Variations. Berlin: Springer-Verlag 1998.

[8] Drakhlin, M. E.: An inner superposition operator in spaces of summable functions (in Russian). Izvestiya Vysh. Uch. Zav. (Matematika) 30 (1986) 5, $18-24$. 
[9] Drakhlin, M. E.: On convergence of sequences of internal superposition operators. Funct. Diff. Equ. Israel Sem. 1 (1993), 83 - 94.

[10] Drakhlin, M. E. and E. Stepanov: Convergence of composition operators and optimal control problems. In: Proc. 2nd World Congr. Nonlin. Anal., Athens (Greece) $10-17$ July 1996 (ed.: V. Lakshmikantam). Oxford: Pergamon Press 1997, Part 1: pp. 505 $512=$ Nonlin. Anal. 30 (1997), $505-512$.

[11] Drakhlin, M. E. and E. Stepanov: Weak convergence of inner superposition operators. Proc. Amer. Math. Soc. 126 (1998), 173 - 179.

[12] Dunford, N. and J. T. Schwartz: Linear Operators. Vol. 1: General Theory. New York: Intersci. Publ 1958.

[13] Halmos, R. P.: Measure Theory (Graduate Texts in Mathematics: Vol. 18). Berlin: Springer-Verlag 1974.

[14] Krasnoselskii, M. A., Zabreiko, P. P., Pustyl'nik, E. I. and P. E. Sobolevskiī: Integral Operators in the Spaces of Summable Functions (in Russian). Moscow: Nauka 1966; English transl.: Noordhoff: Intern. Publ. Leyden 1975.

[15] Pascale, L. De and E. Stepanov: Weak and strong convergence of compositions. Trans. Amer. Math. Soc. (to appear).

[16] Krużík, M. and T. Roubíčck: Explicit characterization of $L^{p}$-Young measures. J. Math. Anal. Appl. 198 (1996), 830 - 843.

[17] Kinderlehrer, D. and P. Pedregal: Characterization of Young measures generated by gradients. Arch. Rat. Mech. Anal. 115 (1991), 329 - 365.

[18] Kinderlehrer, D. and P. Pedregal: Gradient Young measures generated by sequences in Sobolev spaces. J. Geom. Anal. 4 (1994), $59-90$.

[19] Pedregal, P.: Nonlocal variational principles. Nonlin. Anal.: Theory, Methods Appl. 29 (1997), 1379 - 1392.

[20] Sychev, M. A.: A new approach to Young measure theory, relaxation and convergence in energy. Preprint. Pittsburgh: Carnegie Mellon University 1998.

Received 24.11.1998; in revised form 24.06.1999 